\title{
The effect of differentiation agents on inflammatory and oxidative responses of the human neuroblastoma cell line SK-N-SH*
}

\author{
Anna Niewiarowska-Sendo, Katarzyna Patrzalek, Andrzej Kozik and Ibeth Guevara-Lora \\ Department of Analytical Biochemistry, Faculty of Biochemistry, Biophysics and Biotechnology, Jagiellonian University in Krakow, Kraków, Poland
}

\begin{abstract}
Obtaining a suitable experimental cellular model is a major problem for neuroscience studies. Neuroblastoma cell lines have been often applied in studies related to pathological disorders of nervous system. However, in the search for an ideal model, these cells must be differentiated to cancel their tumor character. The subsequent reactions that are caused by differentiation are not always indifferent to the same model. We evaluated the effect of two well known substances, used for SH-N-SK cell line differentiation, retinoic acid (RA) and phorbol-12-myristate-13-acetate (PMA), on the induction of pro-inflammatory and pro-oxidative reactions in these cells. Cells differentiated with PMA were able to produce significantly higher amounts of pro-inflammatory cytokines whereas the release of nitric oxide radicals was similar to that in undifferentiated cells. On the contrary, in RA-differentiated cells no significant changes in cytokine production were observed and the nitric oxide release was decreased. Additionally, the RA-differentiated neuronal model was more sensible to lipopolysaccharide stimulation, producing pro-inflammatory cytokines abundantly. These results suggest that RA-differentiated SH-N-SK cells provide a more suitable experimental model for the study of molecular and cellular mechanisms of the inflammation and oxidative stress in neuronal cells.
\end{abstract}

Key words: Neuroblastoma cell lines, RA, PMA

Received: 24 March, 2015; revised: 16 April, 2015; accepted: 13 May, 2015; available on-line:18 August, 2015

\section{INTRODUCTION}

Many neurological processes are frequently investigated using isolated primary cells of animals. However, primary cultures are difficult to maintain, relatively shortlived and sometimes the isolation procedure is complicated. Moreover, some studies may require sacrificing large numbers of animals. Because living and mature human neurons are not readily available, various cell lines, usually various types of neuroblastoma, can be an alternative for neuroscience research. An immortalized cell line, most frequently used for those purposes is the human neuroblastoma SK-N-SH line that was derived from malignant cells of a bone marrow biopsy taken from a four year-old female neuroblastoma patient. These cells are of neurogenic origin, as evidenced by the high dopamine-b-hydroxylase activity, an enzyme found exclusively in nervous tissues (Biedler et al., 1973). The SK-N-SH cell line is relatively homogeneous and comprises two types of cells, a small neuroblastic type and a flat epithelial one. Nevertheless, the use of this cell line as a tumour model allowed to explore numerous processes related to cancer therapy. However, during development of the stable line of these cells, arrested at an early, immature stage, they retained the ability to differentiate, under appropriate conditions, into neuron-like cells, thus providing a popular human neuronal model. Cell differentiation is often accompanied by the inhibition of certain cellular processes, such as proliferation and migration, and also by changes in cell morphology, such as the growth and elongation of neurites. Several neuroblastoma cell lines, together with SK-N-SH cells, were induced to differentiate with diverse agents, including retinoic acid (RA), phorbol-12-myristate-13-acetate (PMA), brain-derived neurotrophic factor (BDNF) or nerve growth factor (Thiele, 1998). However, the use of some of the differentiating agents can lead to adverse reactions, producing a model of mature neuronal cells, which may possess a basal state unsuitable for further studies. Indeed, apoptotic effects of interferon- $\gamma$ or RA on some neuroblastoma cell lines were reported (Thiele, 1998). On the other hand, some changes in mitochondrial functions in PMA-differentiated human leukemia cell line K652 were also registered (Huang et al., 2014). Thereby, the preparation of a suitable cellular model, obtained through cell differentiation, requires rigorous examinations in order to exclude possible interference in the research to be performed.

In recent years, an increasing number of studies reported participation of chronic inflammation and oxidative stress in the development of neurodegenerative diseases (Khandelwal et al., 2011; Ju et al., 2014; Khalil et al., 2015). Human neuroblastoma cell line SK-N-SH is a neuronal model commonly used in studies of neuronal pathologies, including neurodegenerative disorders (Ajjimaporn et al., 2007, Jayaraj et al., 2013). However, relatively little is known about a potential development of inflammatory and oxidative responses in undifferentiated cells under the influence of differentiating agents. Neurodegenerative diseases are a global problem of the worldwide health, affecting not only the population of

e-mail: ibeth.guevara-lora@uj.edu.pl

* Preliminary report on the same subject has been presented during the 42nd Winter School of Faculty of Biochemistry, Biophysics and Biotechnology, Zakopane 10-14 February 2015

Abbreviations: BDNF, brain-derived neurotrophic factor; BrdU, bromodeoxyuridine; DAN, 2,3-diaminonaphtalene; EF-2, elongation factor 2; FBS, foetal bovine serum; IL-1 $\beta$, interleukin $1 \beta$; IL-6, interleukin 6; LPS, lipopolisaccharide; NeuN, neurospecific nuclear marker; PMA, phorbol-12-myristate-13-acetate; RA, retinoic acid; TNF-a, tumor necrosis factor $a$. 
developing countries but also the countries with a well developed economy. Nowadays, there is a growing interest on genome and proteome research related to these disorders, especially concerning the pathogenesis and development of Parkinson's and Alzheimer's diseases. Therefore, the selection of an adequate model for experiments in vitro, relating to inflammatory and oxidative processes involved in neurodegeneration would be helpful. In our current study, we evaluated two of the most known substances used for cell differentiation RA and PMA - in terms of their ability to triggering the inflammatory response and oxidative stress in SK-N-SH cells. We also analyzed the response of these cells, after differentiation, to a potent pro-inflammatory factor, lipopolisacharide (LPS). At the same time, morphological and biochemical changes related to cell differentiation were examined. Our results suggest that SH-N-SK cells differentiated for 6 days with $5 \mu \mathrm{M}$ RA represent the more suitable experimental model for studying the molecular and cellular mechanisms underlying the pathophysiology of inflammation and oxidative stress in neuronal cells.

\section{MATERIALS AND METHODS}

Chemicals. 2,3-Diaminonaphtalene (DAN), phorbol 12-myristate 13-acetate (PMA), protease inhibitor cocktail, retinoid acid (RA), TRI-reagent and other standard chemicals were supplied by Sigma (USA). Antibiotics, antimycotics, cell culture media, fetal bovine serum (FBS), non-essential amino acids and sodium pyruvate were purchased from Thermo Fisher Scientific (USA).

Cell culture. The human neuroblastoma cell line SK$\mathrm{N}-\mathrm{SH}$ supplied by ATCC (USA) was cultured in MEM medium supplemented with $1 \%$ non-essential amino acids, $1 \mathrm{mM}$ sodium pyruvate, $10 \% \mathrm{FBS}$ and a mixture of antibiotics and antimycotics $(1 \mathrm{U} / \mathrm{ml}$ penicillin, $1 \mu \mathrm{g} / \mathrm{ml}$ streptomycin and $2.5 \mu \mathrm{g} / \mathrm{ml}$ amphotericin B) in a humidified atmosphere containing $5 \% \mathrm{CO}_{2}$ at $37^{\circ} \mathrm{C}$.

Cell differentiation. The neuronal phenotype of SK$\mathrm{N}-\mathrm{SH}$ cells was induced by cell incubation in $1 \% \mathrm{FBS}$ cell culture medium, alone and supplemented with $5 \mu \mathrm{M}$ RA or 25 nM PMA for 6 days with medium change every three days. Cells were also treated with both agents (3 days with $5 \mathrm{mM}$ RA and 3 days with $25 \mathrm{nM}$ PMA). The cells cultured in 10\% MEM were considered as undifferentiated, and these cultured only in 1\% MEM (low FBS medium) served as a control against differentiated cells.

The most commonly measured characteristics of differentiation for neuroblastoma cells is the neurite extension. In order to monitor those changes, cells were photographed in an inverted phase contrast microscope Nikon Eclipse TS100 (Japan) equipped with ToupCam Industrial Digital Camera TP605100A (China) before stimulation and after 3 and 6 days of the treatment. The differentiation index, calculated as the ratio of the dendrite length to the cell diameter, was determined from the cell microscopic images (20× magnification). Measurements were performed in at least 50 cells within randomly chosen fields, using the Image J Software.

Cell proliferation. The proliferation level was analyzed with an immunoenzymatic test, BrdU Cell Proliferation Assay kit (Calbiochem, USA). SK-N-SH cells $\left(6 \times 10^{3}\right)$ were differentiated as mentioned above and on the last day of treatment with differentiating agents a BrdU solution (1:2000) was added for further 24 hours. After that, the BrdU assay was performed according to the manufacturer's instructions. The results were pre- sented as a percentage of BrdU incorporation into cells after differentiation in comparison to undifferentiated cells, the latter assumed represent 100\%. Additionally, total protein concentration in lysates of differentiated and undifferentiated cells was assayed with Lowry method (Lowry et al., 1951).

Caspase activity measurement. Caspase $3 / 7$ activity was assayed with a chemiluminescent method using Caspase-Glo ${ }^{\circledR}$ 3/7 Assay from Promega (USA). Cells $\left(6 \times 10^{3}\right)$ were differentiated in 96-well microplate as mentioned above, and the enzyme activity was measured according to the manufacturer's instructions. Changes in chemiluminescence were analyzed in a microplate reader Synergy H1 from BioTek Instruments (USA). The results were described as the relative enzymatic activity of differentiated cells compared to that of undifferentiated cells.

Gene expression analysis. SK-N-SH cells were differentiated using RA or RA/PMA as described above. For gene expression analysis, the total RNA of undifferentiated and differentiated cells was isolated with TRI-Reagent. Changes of mRNA expression for BDNF, c-myc, interleukin $1 \beta$ (IL-1 $\beta$ ), interleukin 6 (IL-6), neurospecific nuclear marker (NeuN) and tumor necrosis factor $\alpha(\mathrm{TNF}-\alpha)$ were analyzed with a two-step RTPCR procedure. The amount of elongation factor 2 (EF2) mRNA was also determined in each sample for the subsequent quantitative analysis. The cDNA synthesized with M-MLV Reverse Transcriptase kit (Promega, USA), was amplified with Real-time PCR procedure using the SYBR Green kit (Kappa Biosystem, USA) in a Thermocycler 7500 Fast Real-Time PCR System (Life Technologies, USA). Specific primers (at final concentration of $10 \mu \mathrm{M}$ ) were used for PCR reaction. The primer pair sequences for the studied genes are specified in Table 1. The annealing temperature for specific primers during PCR reaction was $58^{\circ} \mathrm{C}$ for c-myc, EF-2 and NeuN, $60^{\circ} \mathrm{C}$ for BDNF and IL- 6 and $62^{\circ} \mathrm{C}$ for $I L-1 \beta$ and TNF-a. The thermal cycler conditions were: $95^{\circ} \mathrm{C}$ for $10 \mathrm{~min}$, followed by $95^{\circ} \mathrm{C}$ for $15 \mathrm{~s}, 15 \mathrm{~s}$ at the corresponding annealing temperature and $72^{\circ} \mathrm{C}$ for $30 \mathrm{~s}$ (40 cycles). Data analysis was undertaken applying a $\Delta C_{t}$ method using a housekeeping gene expression (EF-2) and the 7500 Fast Real-Time PCR Software.

Measurement of nitrite production. A fluorometric assay (Misko et al., 1993) was used to check the influence of differentiating agents on the production of nitric oxide (NO) radicals, measured as nitrite ions. SK-N-SH cells $\left(4 \times 10^{5}\right)$ were seeded on 12 -well microplate, differentiated and then stimulated for 2 hours with $10 \mu \mathrm{g} / \mathrm{ml}$ TNF- $\alpha$. After treatment, $100 \mu \mathrm{l}$ of cell supernatants were mixed with $10 \mu \mathrm{l} 320 \mathrm{mM}$ DAN in 96-well microplate and incubated for 30 minutes at room temperature, protected from light. The reaction was stopped by the addition of $5 \mu \mathrm{l} 2,8 \mathrm{M} \mathrm{NaOH}$ and the emitted fluorescence was measured with the Synergy H1 reader at $\lambda_{\text {ex }}=365$ $\mathrm{nm}$ and $\lambda_{\mathrm{em}}=440 \mathrm{~nm}$.

Measurement of cytokine release. Cytokine concentration in cell supernatants was measured with ELISA kits (IL-1 $\beta$, IL-6 and TNF- $\alpha$ ) from BD Biosciences (USA). Briefly, SK-N-SH cells $\left(5 \times 10^{5}\right)$ were seeded on 12 -well plate and, after differentiation, stimulated for 18 hours with $100 \mathrm{ng} / \mathrm{ml} \mathrm{LPS}$ in the presence of protease inhibitors. Control samples before differentiation, and after differentiation but without LPS stimulation, were also prepared. The released cytokines were assayed according to the manufacturer's instructions. Changes in absorbance were measured at $450 \mathrm{~nm}$ wavelength in PowerWave microplate reader (BioTek Instruments, USA). 
Table 1. Primers sequences used for gene amplification.

\begin{tabular}{|c|c|c|}
\hline Gene & Forward primer & Reverse primer \\
\hline$B D N F$ & 5'ggtaacggcagcagacaaaa' & 5'atccttatgaatcgccagcc3' \\
\hline$c-m y c$ & $5^{\prime}$ tggtcttcccctaccctctcaac3' & 5'gatccagactctgaccttttgcc3' \\
\hline$E F-2$ & 5'gacatcaccaagggtgtgcag3' & 5'gcggtcagcacactggcata3' \\
\hline$I L-1 \beta$ & 5'gatgtctggtccatatgaactg3' & 5'ttgggatctacactctccagc3' \\
\hline$I L-6$ & 5'ccacaagcgccttcggtcca3' & 5'ctgggggtactggggcaggg3' \\
\hline NeuN & 5'gaggacaccttgacttcggt3' & 5'tagtgggaggtgaggtctgc3' \\
\hline$T N F-a$ & 5'tccttcagacaccctcaacc3' & 5'aggccccagtttgaattctt3' \\
\hline
\end{tabular}

Statistical analysis. All values were presented as means \pm standard deviation from at least three experiments and the statistical significance was analyzed by Student's $t$-test.

\section{RESULTS}

\section{Morphological changes of the neuroblastoma cell line SK-N-SH after differentiation}

Three models of SK-N-SH cell differentiation were analyzed in low FBS medium (1\%) with: i) RA, ii) PMA and iii) a combination of RA and PMA (RA/PMA). Results were compared to undifferentiated cells, grown in $10 \%$ FBS medium. Control samples with medium for dif- ferentiation (1\% FBS MEM) were also performed. Changes in cell morphology were observed in every model after just 3 days (not shown). However, they were more evident after 6-day treatment. Each type of differentiation caused the presence of elongated dendrites in cells (Fig. 1D-F), whereas undifferentiated cells proliferated abundantly and did not show any morphological changes (Fig. 1A, 1B). Dendrite growth varied depending on the differentiation method. The shortest dendrites were observed in the cells cultured in low FBS medium (Fig. 1C), whereas the cells treated with $\mathrm{RA}$ and with the combination of RA/ PMA presented the longest dendrites (Fig. 1D and 1F, respectively). The cells differentiated with PMA only showed a relatively high growth of neurites and, additionally, in these samples the amount of cells seems to be lower (Fig. 1E). Those observations are in accordance with the calculated differentiation index for each type of treatment (Fig. 1G). Similar values were obtained for samples with RA, PMA and both RA/PMA, with a 6 -fold increase, after 3 days of differentiation treatment, whereas for the cells treated only with 1\% MEM the differentiation index was definitely lower. After 6-day treatment, the highest values were noticed only for samples with RA and RA/PMA (5-6-fold relatively to undifferentiated cells, that did not present significant changes in their morphology during this incubation time).
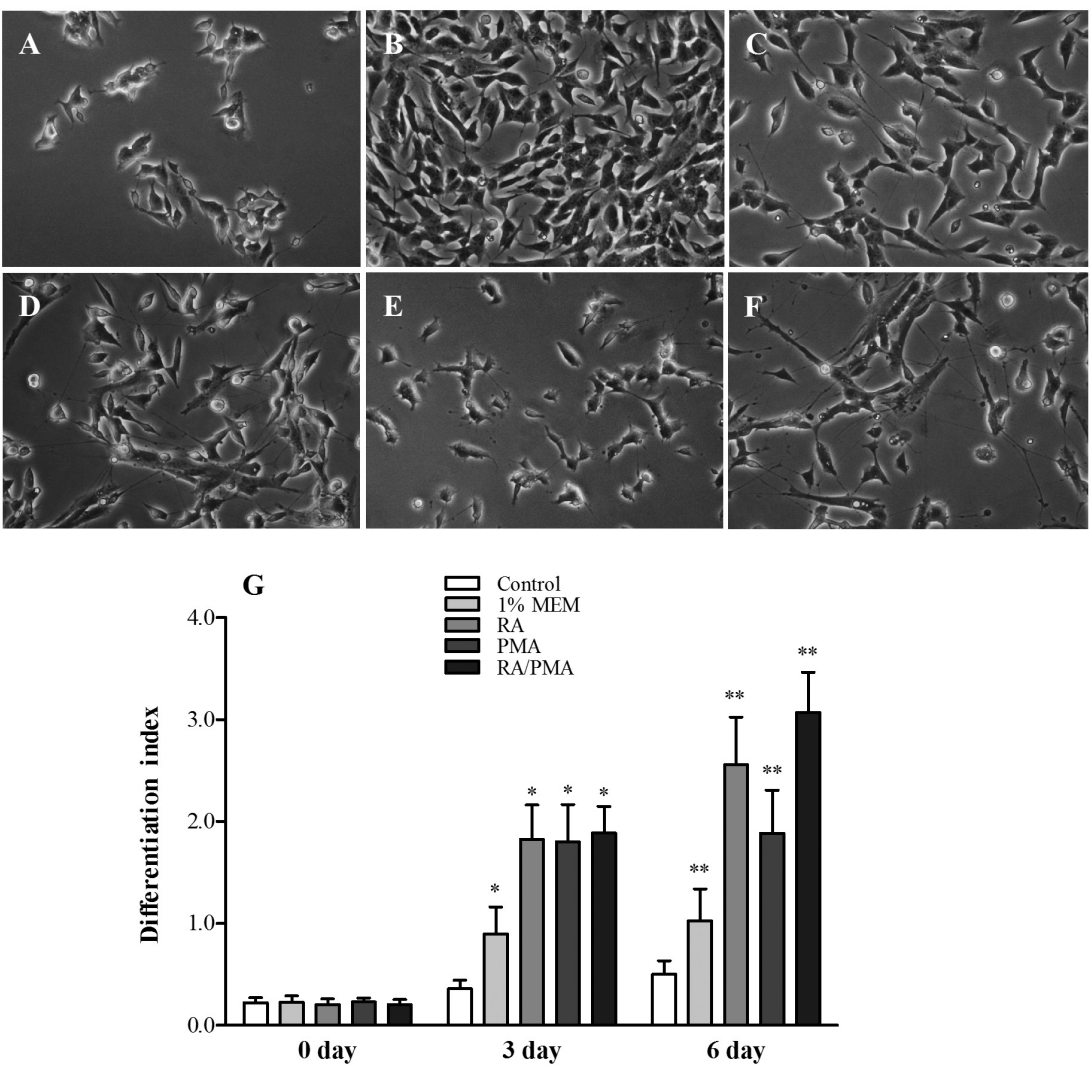

Figure 1. Analysis of the morphological changes of SK-N-SH cells after differentiation.

Morphology of SK-N-SH cells $\left(3 \times 10^{4}\right)$ before (A) and after 6-day treatment with 10\% FBS MEM (B), 1\% MEM (C), $5 \mu M$ RA (D), 25 nM PMA (E) and 3-day treatment with $5 \mu \mathrm{M}$ RA followed by 3-day incubation with $25 \mathrm{nM}$ PMA (F). Cell differentiation level was presented as the differentiation index, which was calculated as the ratio of dendrite length to cell diameter $(\mathbf{G})$. ${ }^{*} P<0.01$ versus undifferentiated cells (control sample) after 3 days, ${ }^{* *} P<0.01$ versus undifferentiated cells after 6 days. 


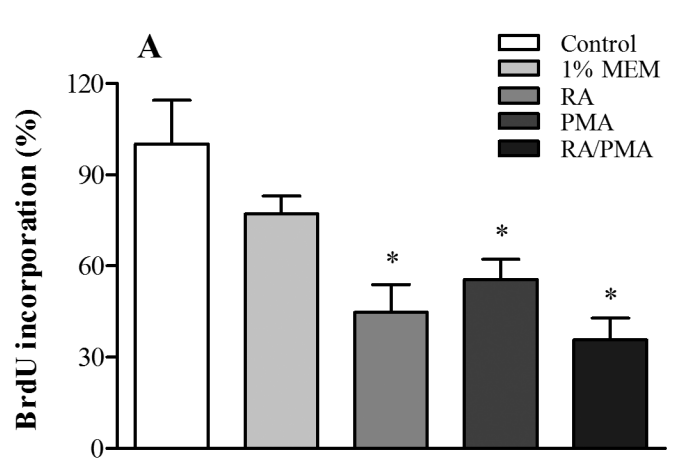

B

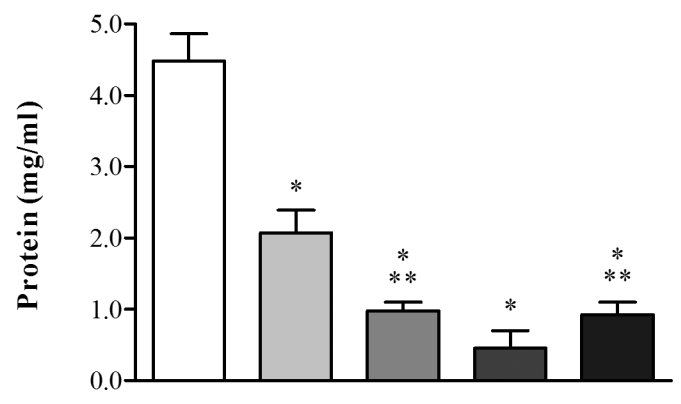

Figure 2. Proliferation of SK-N-SH cells after differentiation. Cells were grown in 10\% MEM, 1\% MEM, $5 \mu \mathrm{M}$ RA, 25 nM PMA or $5 \mu \mathrm{M}$ RA/25 nM PMA for 6 days. Cell proliferation was analyzed by BrdU assay (A) and by measurement of total protein concentration using Lowry method (B). ${ }^{*} P<0.05$ vs. undifferentiated cells in $10 \%$ FBS MEM. ${ }^{* *} P<0.05$ vs. cell cultured in 25 nM PMA.

\section{Cell proliferation of the neuroblastoma cell line SK-N- $\mathrm{SH}$ after differentiation}

The inhibition of cell proliferation is commonly known as the main indicative of cell differentiation. To determine the influence of differentiating agents on cell proliferation, undifferentiated and differentiated SK-NSH cells were checked for BrdU incorporation during $24 \mathrm{~h}$ incubation. The amount of incorporated BrdU into cells varied depending on cell treatment. The BrdU content was similar in cells differentiated by RA and RA/ PMA (about 40\%), whereas treatment with PMA and low FBS medium resulted in $55 \%$ and $77 \%$ incorporation of BrdU, respectively, suggesting a worse differentiation of neuroblastoma into neuronal type cells (Fig. 2A). On the other hand, the analysis of the total protein concentration showed comparable outcomes (Fig. 2B). The highest protein concentration was obtained for samples from undifferentiated cells $(4.48 \mathrm{mg} / \mathrm{ml})$ and cells cultured in $1 \%$ MEM $(2.07 \mathrm{mg} / \mathrm{ml})$, while the lowest values were observed in samples from cells differentiated with PMA $(0.45 \mathrm{mg} / \mathrm{ml})$. Similar amount of total protein was measured in samples with cells differentiated by using $\mathrm{RA}$ and RA/PMA $(0.90 \mathrm{mg} / \mathrm{ml})$.

\section{The effect of differentiation on the neuroblastoma cell line SK-N-SH apoptosis}

The influence of the differentiating agents on SK-N$\mathrm{SH}$ cell apoptosis was analyzed by measuring the caspase $3 / 7$ activity in cells. Increased enzyme activity was observed in samples treated with PMA, both alone or in combination with RA (Fig. 3). In the first case, the percentage of the relative caspase activity reached $280 \%$

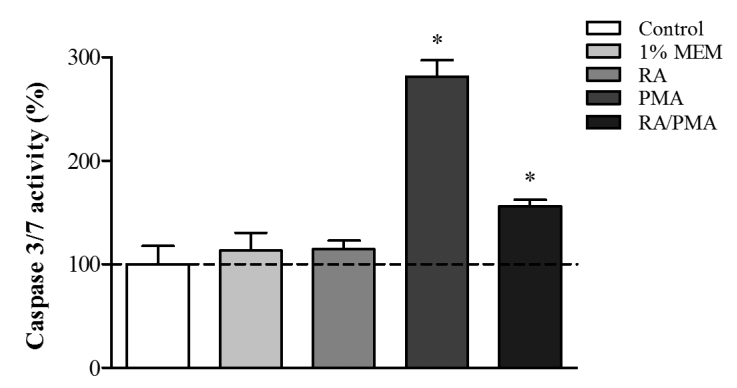

Figure 3. Relative activity of caspase $3 / 7$ in SK-N-SH cells after differentiation.

Cells $\left(6 \times 10^{3}\right)$ were grown in 10\% MEM, 1\% MEM, $5 \mu$ M RA, $25 \mathrm{nM}$ PMA or $5 \mu \mathrm{M}$ RA/25 nM PMA for 6 days. The caspase $3 / 7$ activity was measured with CaspoGlo3 kit and the values obtained are presented as the percentage of changes in chemiluminescence signal in samples as compared to control sample (undifferentiated cells), that was assumed to be $100 \% .{ }^{*} P<0.05$ vs. control cells.

as compared to undifferentiated cells, whereas RA/PMA enhanced cell apoptosis by $55 \%$. No significant differences were observed between undifferentiated cells and cells treated only with RA or 1\% MEM.
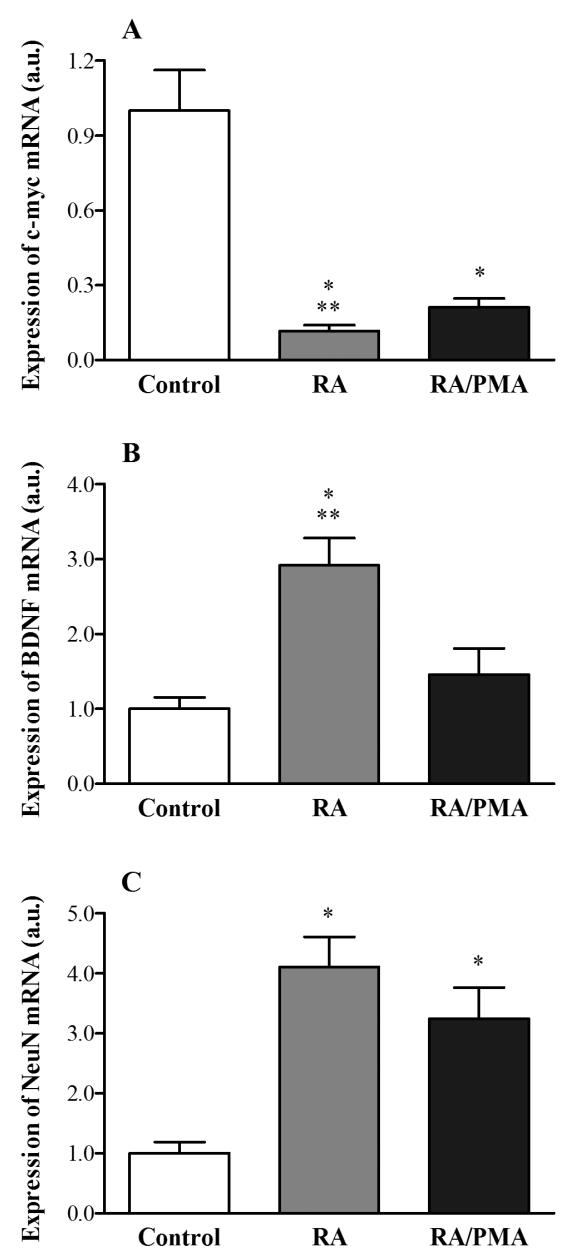

Figure 4. Expression of tumor and neuronal markers after differentiation of SK-N-SH cell line.

Cells $\left(1 \times 10^{6}\right)$ were grown in $10 \%$ MEM, or differentiated in $1 \%$ MEM with $5 \mu \mathrm{M}$ RA or $5 \mu \mathrm{M}$ RA/25 nM PMA for 6 days. The expression for C-myc (A), BDNF (B) and NeuN (C) was analyzed by Real-Time PCR. ${ }^{*} P<0.01$ vs. control sample (undifferentiated cells), ${ }^{*} P<0.05$ vs. RA/PMA sample. 
A

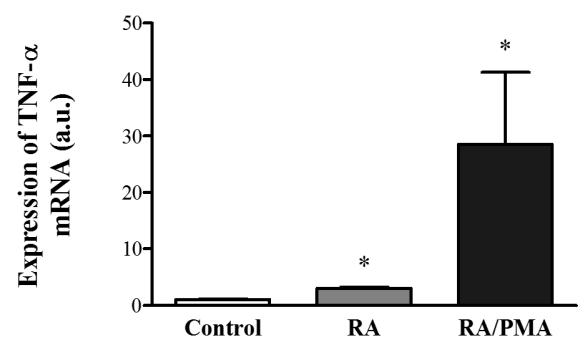

C

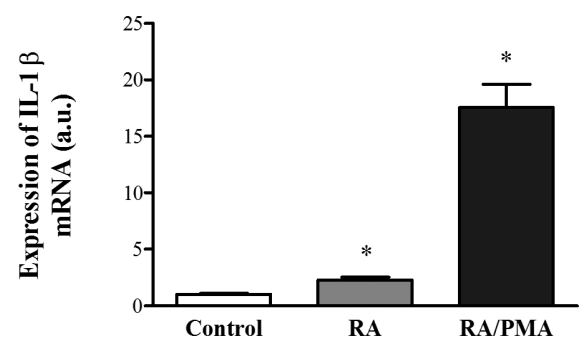

E

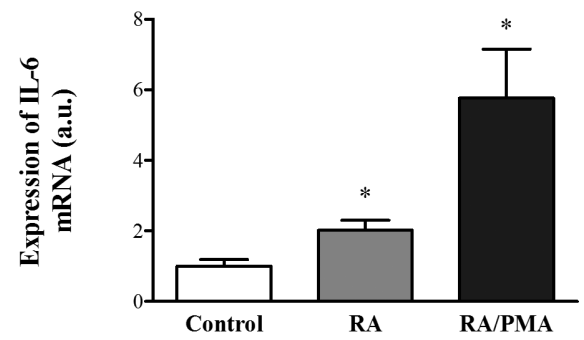

B

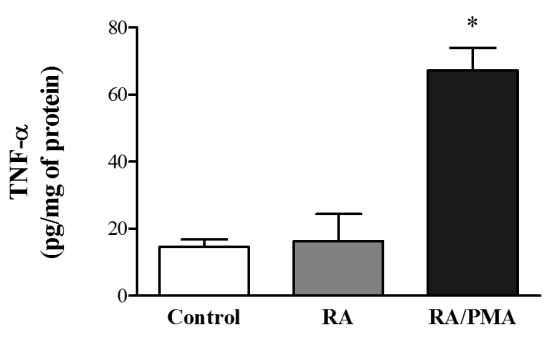

D

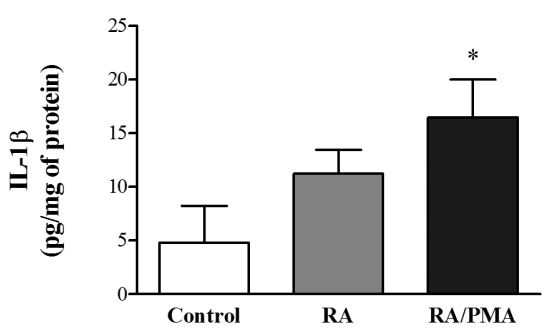

F

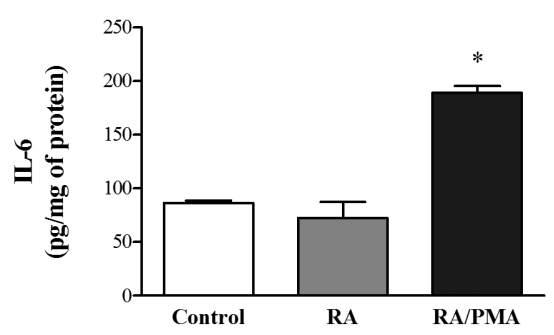

Figure 5. Expression of pro-inflammatory cytokines in SK-N-SH cells after differentiation.

Cells after $80 \%$ confluence were grown in $10 \%$ MEM or differentiated in 1\% MEM with $5 \mu$ M RA or $5 \mu M$ RA/25 nM PMA for 6 days. The mRNA expression and protein production for TNF-a (A, B), IL-1 $\beta$ (C, D) and IL-6 (E, F) were analyzed by Real-Time PCR and ELISA, respectively. ${ }^{*} P<0.05$ versus control sample (undifferentiated cells).

Expression of tumor and neuronal markers after differentiation of the neuroblastoma cell line SK-N-SH

Gene expression for tumor (c-myc) and neuronal (BDNF and NeuN) markers was analyzed to describe the differentiation level of SK-N-SH cells. Real-Time PCR results indicated a significant decrease of c-myc mRNA after differentiation (Fig. 4A). Major changes in oncogene expression were observed after cell differentiation with both RA and RA/PMA, but RA treatment for 6 days caused more accentuated inhibition of c-myc expression than the RA/PMA model. In the case of neuronal markers (Fig. 4B and 4C), both differentiation types resulted in enhanced mRNA expression of BDNF and $\operatorname{NeuN}$, with similar results to these obtained for the cmyc gene. Major effects were observed in cells treated with RA alone.

\section{Proinflammatory changes after SK-N-SH cell differentiation}

Inflammation markers after differentiation of SK-N$\mathrm{SH}$ cells were investigated both at gene expression and

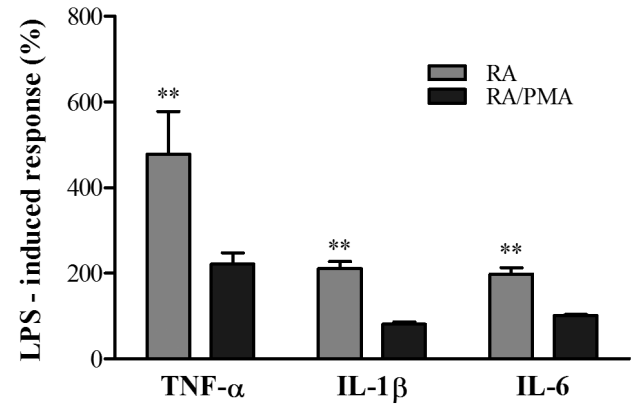

Figure 6. Cytokine production by RA- and RA/PMA-differentiated cells after LPS stimulation.

Cells after $80 \%$ confluence were differentiated with $5 \mu \mathrm{M}$ RA or 5 $\mu \mathrm{M}$ RA/25 nM PMA for 6 days and then stimulated with $100 \mathrm{ng} /$ $\mathrm{ml}$ LPS for $18 \mathrm{~h}$. LPS-induced cytokine production, measured by ELISA, was presented as the percentage of cytokine release after stimulation in comparison to non-stimulated differentiated cells, assumed to be $100 \%$. ${ }^{*} P<0.05$ versus RA/PMA-differentiated cells. 

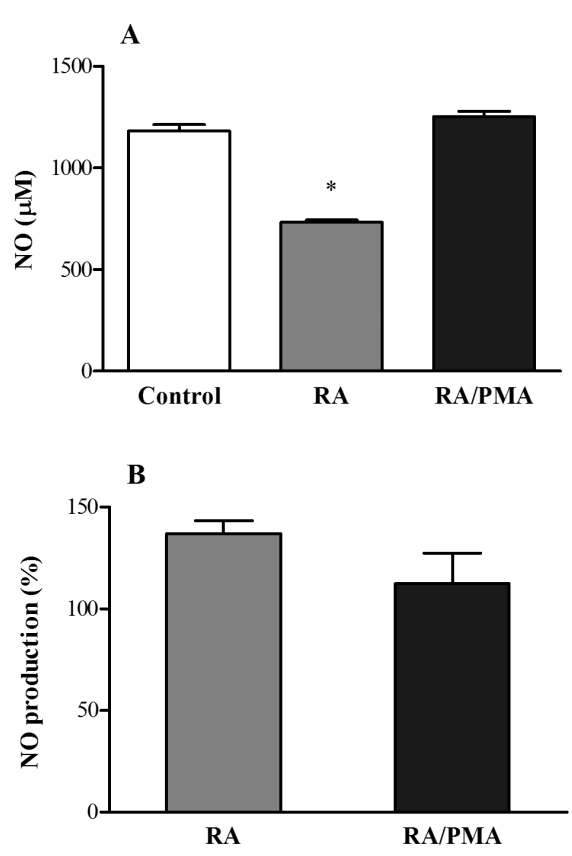

Figure 7. Production of NO in SK-N-SH cells after differentiation. Cells after $80 \%$ confluence were differentiated with $5 \mu \mathrm{M}$ RA or $5 \mu \mathrm{M}$ RA/25 nM PMA for 6 days. The NO concentration in medium from undifferentiated and differentiated cells $(\mathbf{A})$ and the relative change in NO production after 2-hour stimulation with $10 \mu \mathrm{g} / \mathrm{ml}$ TNF-a in comparison to non-stimulated cells (B) was measured with fluorometric assay. ${ }^{*} P<0.01$ versus control sample (undifferentiated cells).

protein production levels. The change in the mRNA expression of cytokines (IL-1 $\beta$, IL-6 and TNF- $\alpha$ ) after differentiation was presented in Fig. 5. Cells treated with RA in combination with PMA showed more significant mRNA expression of all of the studied cytokines (Fig. 5A, 5C and 5E). The RA treatment also caused an augmentation of the amount of cytokine mRNA; however, this increase was definitively lower than in the case of RA/PMA treatment. Those observations were compared with the cytokine release into culture medium assayed with ELISA test. The results corroborated the observed trend in the expression of cytokine mRNA, but only in the case of cells treated with RA/PMA. The level of all studied cytokines was significantly higher (by 363\%, $243 \%$ and $118 \%$, respectively for TNF- $\alpha$, IL- $1 \beta$, and IL$6)$, whereas in cells treated with RA alone their concentration was similar to that observed in undifferentiated cells. Only IL-1 $\beta$ concentration was slightly increased but any statistical significance was observed as compare with untreated cells (Fig 5B, 5D, 5F).

Due to significant changes in cytokine production in the neuron-like cell models studied, LPS stimulation allowed to assess their sensibility to pro-inflammatory agents. The percentage of the LPS-induced cytokine production by these cell models varied significantly (Fig. 6). RA-differentiated cells produced more cytokines in response to LPS stimulation than cells treated with RA/ PMA. The production of TNF- $\alpha$, after LPS stimulation of RA-treated cells was twice higher than in the RA/ PMA model. Similar results were observed for IL-1 $\beta$ and IL- 6 production by RA-differentiated cells after LPS stimulation, indicating a higher cytokine release than that obtained with cells differentiated with RA/PMA, i.e., $211 \%$ and $199 \%$, respectively.
Effect of SK-N-SH cell differentiation on NO production

Since activation of pro-inflammatory response is often associated with stimulation of pro-oxidative processes, in this study we also analyzed the influence of differentiating agents on the modulation of an oxidative marker, the level of $\mathrm{NO}$ radicals. In this regard, the amount of nitrite ions, produced by undifferentiated cells and by cells treated with RA or RA/PMA, was measured. Results obtained by fluorometric assay showed a lower NO production by RA-differentiated cells in comparison to cells treated with RA/PMA, for which NO concentration turned out to be similar to that by the undifferentiated cells (Fig. 7A). Moreover, an analogous study as in the case of pro-inflammatory cytokines was conducted to check the difference between the two cell models to produce NO after TNF- $\alpha$ stimulation. The effect of cytokine stimulation was less significant than that obtained in the case of cytokine release; however, RA-differentiated cells presented a major sensibility to produce $\mathrm{NO}$ than cells treated with RA/PMA (Fig. 7B).

\section{DISCUSSION}

Several studies reported the in vitro differentiation of the SK-N-SH cell line using various agents that promote coordinated biochemical, morphological and ultrastructural changes leading to neuronal phenotypes (Pahlman et al., 1995; Thiele, 1998). SK-N-SH cell line is composed of two types of cells, a small spiny cell (neuronal type) and a large epithelioid cell type (Biedler et al., 1973). Numerous studies reported that, depending on the type of differentiating agents, distinct phenotypes of cells can be obtained. These observations implicate rigorous evaluation of the influence of these agents on cellular signalling pathways, which can be of primordial importance for the selection of an appropriate model for neuroscience. In our studies we checked the influence of the main differentiating factors like RA and PMA on pro-inflammatory and pro-oxidative changes in the widely used SK-N-SH cell line, looking for a reliable cell model of neurodegenerative disease, in which biochemical and molecular changes could be examined during cell degeneration. As expected, our results confirmed the ability of the SK$\mathrm{N}-\mathrm{SH}$ cells to differentiate into neuronal phenotype. Similarly to earlier reports (Lombet et al., 2001; Peng et al., 2015), we also observed morphological changes and elongation of neurites, induced by both RA- and RA/ PMA-treatment after 3 days, that were enhanced after 3 more days (Fig. 1). While the PMA treatment activated cell differentiation, this effect was not as strong as in the case of the previously described procedures. In addition, after further incubation (3 days) a lower amount of cells was observed, suggesting an activation of apoptotic signals, which lead to degeneration of neurites and, in a consequence, to a decrease of the differentiation level. These morphological changes were in accordance with the calculated differentiation index for different studied models.

Moreover, the differentiation processes were also characterized by inhibition of cell proliferation. Our study on BrdU incorporation demonstrated a significant inhibition of cell growth after differentiation treatments (Fig. 2). Every differentiation type - with RA, PMA and a combination RA/PMA - inhibited the proliferation by $50 \%$. These observations corroborated earlier studies, which showed diminished proliferation of neuroblastoma cells by both RA and PMA (Pizzi et al., 2002; Presgraves et al., 2004). Cells cultured in low 
FBS medium without differentiation agents, presented a reduced proliferation rate but this decrease, contrary to earlier reports, was insignificant as compared to undifferentiated cells cultured in a complete medium (Buttiglione et al., 2007). Similar results were obtained from the measurement of total protein concentration in samples after differentiation. Interestingly, the total concentration of protein for the sample treated with PMA was significantly lower than for other differentiated samples. This observation, together with those from the analysis of cell morphology change, allowed to affirm that the PMA treatment caused inhibition of cell proliferation. However, the significant decrease in protein concentration may be also associated with the activation of apoptotic processes, due to the fact that neurite development in these cells was definitely lower and a cell detachment was also observed. Certainly, apoptotic effects have been reported in studies on cell differentiation with PMA (Takada et al., 1999; Chornyy et al., 2007; Itsumi et al., 2014).

In fact, studies on the impact of differentiating factors on cell apoptosis confirmed these affirmations. The results showed that in cells treated with PMA, caspase $3 / 7$ activity increased almost twice (Fig. 3). Even samples differentiated with RA/PMA showed greater activity of this enzyme, although a little smaller than when PMA was only used. On the contrary, samples stimulated with RA presented similar values of this apoptosis marker to those obtained in control cells, both undifferentiated and cultured in low FBS medium. Therefore, we propose that PMA can certainly cause the cell differentiation but at the same time it can activate certain apoptosis pathways which can consequently lead to cell death. Signalling pathways involved in these processes can be associated with protein kinase $C$. This enzyme is an important regulator of cell proliferation, malignant transformation as well as cell apoptosis (Gonzalez-Guerrico et al., 2005). In previous studies, protein kinase $C$ activation in neuroblastoma cells by PMA but not by RA was demonstrated (Bouillon \& Audette, 1993; Soderholm et al., 2001). Bearing in mind these observations, it seems that the use of PMA as a differentiation agent of neuroblastoma cells may results in an inadequate cell model, especially concerning the study of neurodegenerative processes.

Accordingly, following experiments were focused on RA- or RA/PMA-differentiated models, omitting the PMA-differentiated one, which was assumed to be strongly pro-apoptotic due to the large caspase $3 / 7$ activation. At first, the expression of cancerous and neuronal markers after cell differentiation was compared with undifferentiated cells. Our studies showed a decrease in the c-myc mRNA expression, suggesting inhibition of proliferation of differentiated cells by both treatments (RA and RA/PMA). The c-myc is a known oncogene involved in tumor initiation, whose mRNA amplification in studies related to neuroblastoma therapy is recognized as a marker of poor prognosis (Grotzer et al., 2001). Due to intensive $c-m y c$ gene expression in the neuronal type of SK-N-SH cells, this oncogene was selected as a marker of the tumor inhibition (Sadee et al., 1987). In addition, previous reports demonstrated that RA and PMA were able to inhibit the expression of this gene (Pahlman et al., 1991, Shi et al., 2010). In our study we also showed, that differentiating treatment, with RA alone or in combination with PMA, caused an essential decrease on c-myc mRNA in comparison to undifferentiated cells. However, a higher, significant effect was observed after RA treatment (Fig. 4A). These results support our earlier assumption, that the RA-differentiation of SK-N-SH cell line seems to be more advantageous than the use of
PMA. A similar conclusion can be based on the analysis of the appearance of neuronal markers after differentiation. Both treatments, with RA as well as with RA/ PMA were able to induce strong mRNA expression of neuronal markers, BDNF and NeuN as compared to undifferentiated cells (Fig. 4B and 4C). These observations confirmed previous studies in which enhanced $B D N F$ and $N e u N$ expression was developed after differentiation in several neuroblastoma cells (Palhman et al., 1995; Feng et al., 2001; Pizzi et al, 2002; Cheung et al., 2009; Agholme et al., 2010). However, we noticed a significant difference in the amount of BDNF mRNA in the studied models in favour of the RA-differentiation model, arguing for their superiority.

In the pathogenesis of several disorders involving nervous tissues, including neurodegenerative diseases, the chronic inflammation plays an important role (Khandelwal et al., 2011). Additionally, the development of chronic inflammation is associated with impaired balance between production and degradation of cytokines. In this study, we checked the effect of differentiation agents on the production of the most important pro-inflammatory cytokines - TNF- $\alpha$, IL-1 $\beta$ and IL- 6 - by neuroblastoma cell line SK-N-SH. All analyzed cytokines were strongly up-regulated at gene expression level, and a potent protein release by cells after RA/PMA treatment was observed, whereas the use of RA as the differentiation factor caused a more attenuated effect that was insignificant in terms of protein release (Fig 5). Studies on cytokine production after RA and PMA differentiation, performed in other types of cells, showed similar results. In experiments carried out on the murine macrophage cell line J774A.1, mRNA expression of TNF- $\alpha$ and IL$1 \beta$ was increased by RA, but this effect was not translated to protein release by cells (Mathew \& Sharma, 2000). In turn, phorbol esters caused enhanced expression and translation of $\mathrm{TNF}-\alpha$ in a human malignant histiocytosis cell line. However, IL-1 $\beta$ protein could not be produced, suggesting a different pathway for the regulation of the synthesis of these cytokines (Gogusev et al., 1996). The most interesting results were associated with the study on the sensitivity of the differentiated cell models to induce inflammatory response after LPS stimulation (Fig. 6). We compared the cytokine release by SK-N-SH cells differentiated with RA or RA/PMA, obtaining unambiguous results, which showed a stronger response of cells treated with RA to inflammatory stimuli. Perhaps this may be related to the anti-inflammatory effect attributed to RA (Bai et al., 2009; Gu et al., 2010), whereas PMA treatment is associated with a pro-inflammatory response (Chiang et al., 2014; Zhang et al., 2013). In fact, a great production of pro-inflammatory cytokines was documented after LPS stimulation in others neuronal models. One of the most popular models is comprised by embryonic PC12 cells isolated from a pheochromocytoma of rat adrenal medulla. These cells, after differentiation with nerve growth factor, were able to release enormous amount of cytokines, such as TNF- $\alpha$ and IL$1 \beta$ after LPS treatment (Muchhala \& Benzeroual, 2012; Yagnik \& Benzeroual, 2013). Hence, we can assume that the large production of cytokines, reached by RA-differentiated cells in response to LPS, argues for the high effectiveness of this differentiating agent as compared to phorbol esters in the preparation of suitable neuronal model. Therefore, these observations allow us to suggest once again the superiority of RA-treatment for differentiation of SK-H-SN cells.

Inflammatory response is strictly associated with disturbance of the oxidant-antioxidant balance. Abundant 
increase of free radicals in cells initiates the inflammatory processes that lead to cell death. The production of nitric oxide radical — one of the most popular free radicals involved in neurological disorders (Emerit et al., 2004) was analyzed in order to prove the influence of RA and PMA on oxidative stress during differentiation processes. The results indicated a decreased production of NO by RA-treated cells, whereas the NO level in cells differentiated with RA/PMA was similar to that in control cells (Fig. 7A). Earlier studies documented a similar effect of RA in J774A.1 cells, a murine macrophage cell line and in human neuroblastoma cell line SH-5YSY (Mathew \& Sharma, 2000; Cheung et al., 2009). The opposite effect induced by PMA was also shown (Hortelano et al, 1992; Kawase et al. 1998). Moreover, a pro-oxidative effect of PMA in human fibrosarcoma cells, through the stimulation of the production of reactive oxidative species, was proposed (Kim et al., 2013).

Therefore, our observations are in accordance with the studies above described. For this reason, the ability of differentiated cells to produce $\mathrm{NO}$ after $\mathrm{TNF}-\alpha$ stimulation was additionally studied. The PMA-treated cells showed no changes in NO production after stimulus, whereas cells differentiated with RA presented only an attenuated increase of $\mathrm{NO}$ release after $\mathrm{TNF}-\alpha$ incubation for 2 hours (Fig. 7B). Despite the growing tendency in cytokine-induced NO release, observed in RA-differentiated cells, these results did not present a significant difference from those from PMA-treated cells. Several studies performed with differentiated PC12 cells have showed an elevated NO concentration in medium after stimulation with LPS alone or in combination with TNF- $\alpha$ (Heneka et al., 1998; Ansari et al., 2011; Yagnik \& Benzeroual, 2013). Moreover, an enhanced production of ROS or changes in the activity of anti-oxidative enzymes were also detected after stimulation of PC12 cells with cytokines (Khodagholi \& Tussi, 2011; Chen et al., 2013). Taking into account these studies, we also expected a decisive increase of $\mathrm{NO}$ release by $\mathrm{SK}-\mathrm{N}-\mathrm{SH}$ cells after treatment with $\mathrm{TNF}-\alpha$, especially in RA-differentiated cells. Unfortunately, in our study we did not observe significant changes in NO production. However, it should be noted that PMA differentiation of these cells resulted in a production of large amount of $\mathrm{TNF}-\alpha$, which may, in turn, activate pathways, causing an increased free radical production (e.g., NO) before an exogenous TNF- $\alpha$ stimulation. While the results obtained with PMA-treated cell can be explained in this way, the slight NO production observed after TNF- $\alpha$ stimulation in RA-differentiated cells is difficult to explicate. In this case, RA did not affect significantly the cytokine release. Additional studies on oxidative stress related to differentiation treatments, e.g., including the analysis of different markers, could provide interesting conclusions.

Our results confirm that pathways of cell differentiation may be of crucial importance for obtaining a neuronal model and that RA allowed to achieve a more appropriate human cell model for inflammatory studies in neuroscience. It should be emphasized here, that the observations on differentiation level and the pro-inflammatory responses achieved in our studies cannot be attributed to cell line heterogeneity. We performed experiments with SK-N-SH cells including a high percentage of neuronal phenotype (greater than $95 \%$ as it was confirmed under microscopy observation). Hence, the detected differences in studied models should be attributed to the effect of differentiation factors on cell functions, especially on the controlling of signaling pathways. Indeed, Xie at al. (2010) described the influence of various differentiation agents on the SH-5YSY neuroblastoma cell line with the aim to obtain a cellular model of Parkinson's disease, suggesting varied susceptibility of those models to neurotoxins. In conclusion, we demonstrated that RA-treated SK-N-SH cells may constitute an excellent model to study inflammatory processes associated with disorders of nervous tissues, because cancer cells were able to transform into neuron-like cells, without apoptotic signals and exhibiting greater sensitivity to proinflammatory stimuli.

\section{Declaration of Interest}

The authors report no conflicts of interest.

\section{REFERENCES}

Agholme L, Lindstrom T, Kagedal K, Marcussona J, Hallbeck M (2010) An in vitro model for neuroscience: differentiation of SHSY5Y cells into cells with morphological and biochemical characteristics of mature neurons. J Alzheimers Dis 20: 1069-1082.

Ajjimaporn A, Phansuwan-Pujito P, Ebadi M, Govitrapong P (2007) Zinc protects SK-N-SH cells from methamphetamine-induced alpha-synuclein expression. Neurosci Lett 419: 59-63.

Ansari N, Khodagholi F, Amini M, Shaerzadeh F (2011) Attenuation of LPS-induced apoptosis in NGF-differentiated PC12 cells via NF$x \mathrm{~B}$ pathway and regulation of cellular redox status by an oxazine derivative. Biochimie 93: 899-908.

Bai A, Lu N, Guo Y, Liu Z, Chen J, Peng Z (2009) All-trans retinoic acid down-regulates inflammatory responses by shifting the Treg/ Th17 profile in human ulcerative and murine colitis. J Leukoc Biol 86: 959-969.

Biedler JL, Helson L, Spengler BA (1973) Morphology and growth, tumorigenicity, and cytogenetics of human neuroblastoma cells in continuous culture. Cancer Res 33: 2643-2652.

Bouillon M, Audette M (1993) Transduction of retinoic acid and gamma-interferon signal for intercellular adhesion molecule-1 expression on human tumor cell lines: evidence for the late-acting involvement of protein kinase C inactivation. Cancer Res 53: 826-832.

Buttiglione M, Vitiello F, Sardella E, Petrone L, Nardulli M, Favia P, d'Agostino R, Gristina R (2007) Behaviour of SH-SY5Y neuroblastoma cell line grown in different media and on different chemically modified substrates. Biomaterials 28: 2932-2945.

Chen W, Li X, Jia L-Q, Wang J, Zhang L, Hou D, Wang J, Ren L (2013) Neuroprotective activities of catalpol against CaMKII-dependent apoptosis induced by LPS in PC12 cells. Br J Pharmacol 169: 1140-1152.

Cheung YT, Lau WK, Yu MS, Lai CS, Yeung SC, So KF, Chang RC (2009) Effects of all-trans-retinoic acid on human SH-SY5Y neuroblastoma as in vitro model in neurotoxicity research. Neurotoxicology 30: $127-135$.

Chiang KC, Tsui KH, Chung LC, Yeh CN, Chen WT, Chang PL, Juang HH (2014) Celastrol blocks interleukin-6 gene expression via downregulation of NF- $x \mathrm{~B}$ in prostate carcinoma cells. PLoS One 9: e93151.

Chornyy S, Parkhomenko J, Chorna N (2007) Thiamine deficiency caused by thiamine antagonists triggers upregulation of apoptosis inducing factor gene expression and leads to caspase 3-mediated apoptosis in neuronally differentiated rat PC-12 cells. Acta Biochim Pol 54: 315-322.

Emerit J, Edeas M, Bricaire F (2004) Neurodegenerative diseases and oxidative stress. Biomed Pharmacother 58: 39-46.

Feng X, Jiang H, Baik JC, Edgar C, Eide FF (2001) BDNF dependence in neuroblastoma. J Neurosci Res 64: 355-363.

Gogusev J, Barbey S, Nezelof C (1996) Regulation of TNF-alpha and IL-1 gene expression during TPA-induced differentiation of "Malignant histiocytosis" DEL cell line $\mathrm{t}(5 ; 6)$ (q35:p21). Anticancer Res 16: $455-460$.

Gonzalez-Guerrico AM, Meshki J, Xiao L, Benavides F, Conti CJ, Kazanietz MG (2005) Molecular mechanisms of protein kinase Cinduced apoptosis in prostate cancer cells. J Biochem Mol Biol 38: 639-645.

Grotzer MA, Hogarty MD, Janss AJ, Liu X, Zhao H, Eggert A, Sutton LN, Rorke LB,. Brodeur GM, Phillips PC (2001) MYC messenger RNA expression predicts survival outcome in childhood primitive neuroectodermal tumor/medulloblastoma. Clin Cancer Res 7: 24252433.

Gu B, Miao J, Fa Y, Lu J, Zou S (2010) Retinoic acid attenuates lipopolysaccharide-induced inflammatory responses by suppressing TLR4/NF-kappaB expression in rat mammary tissue. Int Immunopharmacol 10: 799-805. 
Heneka MT, Löschmann PA, Gleichmann M, Weller M, Schulz JB, Wullner U, Klockgether T (1998) Induction of nitric oxide synthase and nitric oxide-mediated apoptosis in neuronal PC12 cells after stimulation with tumor necrosis factor-a/lipopolysaccharide $J$ Neurochem 71: 88-94.

Hortelano S, Genaro AM, Bosca L (1992) Phorbol esters induce nitric oxide synthase activity in rat hepatocytes. Antagonism with the induction elicited by lipopolysaccharide. J Biol Chem 267: $24937-$ 24940.

Huang R, Zhao L, Chen H, Yin RH, Li CY, Zhan YQ, Zhang JH, Ge CH, Yu M, Yang XM (2014) Megakaryocytic differentiation of $\mathrm{K} 562$ cells induced by PMA reduced the activity of respiratory chain complex IV. PLOS ONE 9: e96246.

Itsumi M, Shiota M, Yokomizo A, Takeuchi A, Kashiwagi E, Dejima T, Inokuchi J, Tatsugami K, Uchiumi T, Naito S (2014) PMA induces androgen receptor downregulation and cellular apoptosis in prostate cancer cells. J Mol Endocrinol 53: 31-41.

Jayaraj RL, Tamilselvam K, Manivasagam T, Elangovan N (2013) Neuroprotective effect of CNB-001, a novel pyrazole derivative of curcumin on biochemical and apoptotic markers against rotenoneinduced SK-N-SH cellular model of Parkinson's disease. $J \mathrm{Mol} \mathrm{Neu-}$ rosci 51: 863-870.

Ju TC, Chen HM, Chen YC, Chang CP, Chang C, Chern Y (2014) AMPK- $\alpha 1$ functions downstream of oxidative stress to mediate neuronal atrophy in Huntington's disease. Biochim Biophys Acta 1842: 1668-1680.

Kawase T, Orikasa M, Oguro A, Burns DM (1998) Up-regulation of inducible nitric oxide (NO) synthase and NO production in HL-60 cells stimulated to differentiate by phorbol 12-myristate 13-acetate plus 1,25-dihydroxyvitamin D3 is not obtained with dimethylsulfoxide plus 1,25-dihydroxyvitamin D3. Calcif Tissue Int 63: 27-35.

Khalil WK, Assaf N, ElShebiney SA, Salem NA (2015) Neuroprotective effects of bee venom acupuncture therapy against rotenone-induced oxidative stress and apoptosis. Neurochem Int 80: 79-86.

Khandelwal PJ, Herman AM, Moussa CE (2011) Inflammation in the early stages of neurodegenerative pathology. I Neuroimmunol 238: $1-11$.

Kim A, Im M, Yim NH, Jung YP, Ma JY (2013) Aqueous extract of Bambusae Caulis in Taeniam inhibits PMA-induced tumor cell invasion and pulmonary metastasis: suppression of $\mathrm{NF}-\varkappa \mathrm{B}$ activation through ROS signaling. PLoS One 8: e78061.

Khodagholi F, Tusi SK. (2011) Stabilization of Nrf2 by tBHQ prevents LPS-induced apoptosis in differentiated PC12 cell. Mol Cell Biochem 354: 397-112.

Lombet A, Zujovic V, Kandouz M, Billardon C, Carvajal-Gonzalez S, Gompel A, Rostene W (2001) Resistance to induced apoptosis in the human neuroblastoma cell line SK-N-SH in relation to neuronal differentiation. Role of Bcl-2 protein family. Eur J Biochem 268: 1352-1362.

Lowry OH, Rosebrough NJ, Farr AL, Randall RJ (1951) Protein measurement with the Folin phenol reagent. J Biol Chem 193: 265-275.

Mathew JS, Sharma RP (2000) Effect of all-trans-retinoic acid on cytokine production in a murine macrophage cell line. Int I Immunopbarmacol 22: 693-706.
Muchhala KS, Benzeroual KE (2012) Pentoxifylline suppressed LPSinduced inflammatory and apoptotic signaling in neuronal cells. $A d v$ Biosci Biotech 3: 371-379.

Misko TP, Schilling RJ, Salvemini D, Moore WM, Currie MG (1993) A fluorometric assay for the measurement of nitrite in biological samples. Anal Biochem 214: 11-16.

Pahlman S, Meyerson G, Lindgren E, Schalling M, Johansson I (1991) Insulin-like growth factor I shifts from promoting cell division to potentiating maturation during neuronal differentiation. Proc Natl Acad Sci USA 88: 9994-9998.

Pahlman S, Hoehner JC, Nanberg E, Hedborg F, Fagerstrom S, Gestblom C, Johansson I, Larsson U, Lavenius E, Ortoft E, Soderholm $\mathrm{H}$ (1995) Differentiation and survival influences of growth factors in human neuroblastoma. Eur J Cancer 31A: 453-458.

Peng H, Ke XX, Hu R, Yang L, Cui H, Wei Y (2015) Essential role of GATA3 in regulation of differentiation and cell proliferation in SKN-SH neuroblastoma cells. Mol Med Rep 11: 881-886.

Pizzi M, Boroni F, Bianchetti A, Moraitis C, Sarnico I, Benarese M, Goffi F, Valerio A, Spano P (2002) Expression of functional NR1/ NR2B-type NMDA receptors in neuronally differentiated SK-N-SH human cell line. Eur J Neurosci 16: 2342-2350.

Presgraves SP, Ahmed T, Borwege S, Joyce JN (2004) Terminally differentiated SH-SY5Y cells provide a model system for studying neuroprotective effects of dopamine agonists. Neurotox Res 5: 579-598.

Sadee W, Yu C, Richards ML, Preis PN, Schwab MR, Brodsky FM, Biedler JL (1987) Expression of neurotransmitter receptors and myc protooncogenes in subclones of a human neuroblastoma cell line. Cancer Res 47: 5207-5212.

Shi SL, Liang Y, Li QF, Liu QR, Jing GJ, Wang SY, Zhang XY, Wu FY (2010) Localization of nucleophosmin in nuclear matrix and changes in its expression during the differentiation of human neuroblastoma induced by retinoic acid. I Cell Biochem 111: 67-74.

Soderholm H, Olsson A, Lavenius E, Ronnstrand L, Nanberg E (2001) Activation of Ras, Raf-1 and protein kinase $C$ in differentiating human neuroblastoma cells after treatment with phorbol ester and NGF. Cell Signal 13: 95-104.

Takada Y, Hachiya M, Osawa Y, Hasegawa Y, Ando K, Kobayashi Y, Akashi M (1999) 12-O-tetradecanoylphorbol- 13-acetate-induced apoptosis is mediated by tumor necrosis factor alpha in human monocytic U937 cells. J Biol Chem 274: 28286-28292.

Thiele CJ (1998) Neuroblastoma. In Human Cell Culture Masters J, ed, vol 1, pp 21-53. Kluwer Academic Publishers, Lancaster, UK.

Xie H-r, Hu L-s, Li G-y (2010) SH-SY5Y human nueroblastoma cell: in vitro cell model of dopaminergic neurons in Parkinson's disease. Chin Med J 123: 1086-1092.

Yagnik RM, Benzeroual KE (2013) Tigecycline prevents LPS-induced release of pro-inflammatory and apoptotic mediators in neuronal cells. Toxicol In Vitro 27: 686-693.

Zhang G, Liu X, Wang C, Qu L, Deng J, Wang H, Qin Z (2013) Resolution of PMA-induced skin inflammation involves interaction of IFN- $\gamma$ and ALOX15. Mediators Inflamm 2013: 930124. 